\section{Zinc supplementation enhances linear growth in school-aged children: A randomized controlled trial}

\section{Sanguansak Rerksuppaphol, ${ }^{1}$ Lakkana Rerksuppaphol²}

1Department of Pediatrics, 2Department of Preventive Medicine, Faculty of Medicine, Srinakharinwirot University, Thailand

\begin{abstract}
Zinc supplementation in children in endemic area of zinc deficiency had been reported of growth enhancement; however, the results were inconsistence. This study aimed to evaluate the efficacy of zinc supplementation on growth of school-aged children. A randomized double-blinded controlled trial was conducted in 140 Thai school children. Placebo or zinc bisglycinate (15 mg element zinc) were taken on school day for 6 months. Pre- and posttreatment anthropometric parameters were recorded. Changes in height from the baseline was considered as the primary outcome whereas, changes in other anthropometric parameters were considered as the secondary outcomes. The mean age was 8.9 years; baseline anthropometric data were not significant different between groups. At the end of study, children in zinc group had significantly higher gain in height $(5.6 \pm 2.4 \mathrm{vs}$ $4.7 \pm 1.4 \mathrm{~cm}$, respectively; $\mathrm{P}=0.009)$ and height Z-score $(0.45 \pm 0.37$ vs $0.37 \pm 0.27$, respectively; $\mathrm{P}=0.048$ ) than children in control group. No significant differences of other anthropometric parameters were observed. In conclusion, zinc supplementation increased linear growth in Thai schoolaged children over a 6-month period but it had no effects on other anthropometric parameters.
\end{abstract}

\section{Introduction}

Zinc $(\mathrm{Zn})$ is an essential trace mineral necessary for the proper functioning of multiple aspects of metabolism. It is present in numerous metalloenzymes, including major enzyme-classes. Chelation of Zinc with amino acids cysteine and histidine forms zinc fingers, which are important domains involved in in mRNA transcription. ${ }^{1}$ Zinc also regulates the expression of the metallothionein gene, apoptosis and synaptic signaling. Therefore, zinc plays a critical role in many biochemical pathways and physio- logical processes in the body including cell growth, cell differentiation and metabolism and reproduction. ${ }^{2}$

Zinc deficiency is characterized by growth retardation, loss of appetite, and impaired immune function. In severe cases, zinc deficiency can result in hair loss, diarrhea, delayed sexual maturation, impotence, hypogonadism in males, and eye and skin lesions.3,4 Zinc deficiency in children is responsible for an increased risk of limited growth, developing diarrheal diseases and acute lower respiratory tract infections.5-7 Zinc deficiency is common in the developing countries. ${ }^{8-10}$ Thailand is classified as a medium risk country for zinc deficiency, since the prevalence of zinc deficiency is estimated to be more than $40 \%$ in Thai population. ${ }^{11}$

Currently, the results of supplementing zinc and its impact on health are not clear. Although several studies and meta-analyses are available on this topic, the differences in the study protocols make it difficult to establish the role of zinc supplementation on health. ${ }^{12,13}$ However, there is a general consensus that benefits of zinc supplementation in areas of high deficiency outweigh the harms. ${ }^{14}$ There are few studies investigating the effect of zinc supplementation in school-aged children. There are very few studies conducted on the effect and outcomes of zinc supplementation in Thailand, despite Thailand being an endemic area of zinc deficiency among population. In this study, we aimed to establish the effect of zinc supplementation on growth of schoolaged children.

\section{Materials and Methods}

\section{Study design and subjects}

This study was a double-blind randomized controlled trial conducted in a public school in Ongkharuck district in a central Thailand from June-December 2013. Healthy schoolchildren studying in grade 16 were assessed for eligibility to participate in the study. Children with a known history of chronic illnesses such as chronic liver or renal diseases, congenital heart abnormalities, chronic respiratory disorders, neurological conditions and disorders, behavioral or psychiatric problem or diabetes mellitus were excluded from the study. Children regularly taking vitamin or mineral supplements and those known to be allergic to vitamin and mineral supplements were also excluded from the study. The study was conducted in compliance to the principles of the Declaration of Helsinki. The study protocol was approved by the ethics com-
Correspondence: Sanguansak Rerksuppaphol, Department of Pediatrics, Faculty of Medicine, Srinakhariwirot University, 62 Mo 7, RangsitNakorn Nayok Rd., Nakorn Nayok 26120, Thailand.

Tel.: +662.9058055 - Fax:+663.7395275

E-mail: sanguansak_r@hotmail.com

Acknowledgements: The authors would thank all children and their parents who participated in the study and the many people that assisted with the research project.

Key words: Child, dietary supplement, growth, zinc

Contributions: the authors contributed equally.

Conflict of interest: the authors declare no conflict of interest.

Funding: the study was supported by grants from Srinakharinwirot University, Thailand.

Received for publication: 1 July 2017.

Revision received: 31 August 2017.

Accepted for publication: 1 September 2017.

This work is licensed under a Creative Commons Attribution NonCommercial 4.0 License (CC BY-NC 4.0).

CC Copyright S. Rerksuppaphol

and L. Rerksuppaphol, 2017

Licensee PAGEPress, Italy

Pediatric Reports 2017; 9:7294

doi:10.4081/pr.2017.7294

mittee of the Faculty of Medicine, Srinakharinwirot University. Before enrolment, a written informed consent and assent was obtained from parents or legal guardians and children, respectively. Children were allowed to withdraw from the study at any time and the reason for withdrawal was recorded. The enrolled children were randomized to the zinc or placebo group by a computerized program (GraphPad QuickCals) using a block of two by a statistical consultant who was not involved in the implementation phase of the study. The investigators, teachers, children and parents were masked to the intervention. The code to the randomization sequence was opened only after study completion. This study is registered into the Thai Clinical Trials Registry (Trial number: TCTR20130000003).

\section{Intervention}

On each school day, children in the zinc group were given a sachet of $15 \mathrm{mg}$ elemental zinc in form of zinc bisglycinate powder (Qualimed, Thailand) which was dissolved 
in a glass of plain water before consumption for a period of 6 months. The percentage of zinc in zinc bis-glycinate is $25 \%$. The supplementation powder was not provided during school holidays or in school-absence days. Children in placebo group were given placebo powder which was prepared by the same company in the identical package, color and taste. Placebo solution was prepared in a similar manner by dissolving in water. Children and parents were instructed not to take any vitamin or mineral supplements during the study period.

\section{Data collection and monitoring}

Baseline demographic characteristics including weight, height, waist and hip circumferences, mid-upper arm circumference (MUAC), skin fold thickness and body fat composition were measured by a trained staff. Weight was measured to the nearest 100 grams using an electronic scale (Tanita Body Composition Analyzer, Model BF680W, Tokyo, Japan). Height was measured to the nearest $0.1 \mathrm{~cm}$ using a height rod (Seca, Model 220, Hamburg, Germany). To compare individual growth status of children of different age and sex, weight for age Z-score (WAZ) and height for age Z-score (HAZ) were calculated using the Thai child growth standard. ${ }^{15}$ Waist circumference was measured at the midpoint between the lower costal margin and the top of iliac crest while the subject was in the standing position using a non-stretch tape. Hip circumference was measured to the nearest $0.1 \mathrm{~cm}$ in the standing position at the maximum circumference over the buttock. MUAC was measured at the mid-point between olecranon process and the acromion process of the left arm. Biceps and triceps skinfold thickness were measured over the biceps and triceps muscle, respectively, at the same level as mid arm circumference measurement using a Lange skinfold caliper. Subscapular skinfold thickness was measured at the lower angle of the scapula of a relaxed left arm using the same caliper. Supra-iliac skinfold thickness was measured at the mid axillary line just above iliac crest on the left side. Body fat composition was measured by bioelectric impedance analysis (Tanita Body Composition Analyzer, Model BF-680W, Tokyo, Japan). Weight and height were measured at one-month intervals, while the waist and hip circumferences, MUAC, skinfold thicknesses and body fat composition were measured at the end of study by the same staff using same scales. Adverse events and compliance with treatment were monitored at each visit based on parent reports and drug counts, respectively.

\section{Outcome measurements}

The primary outcome was the improvement of linear growth at the end of study from baseline. Linear growth was assessed by individual gross height gain and improvement of HAZ from baseline. Changes of other anthropometric characteristics including weight, circumferences and skinfold thickness were considered as secondary outcomes.

\section{Sample size}

To provide the study with a statistical power of $80 \%$ and a two-sided type I error of 0.05 to detect a $15 \%$ increase in height in the treatment group from the average height gain in the control group of $4.5 \mathrm{~cm}$ (SD $1.2)^{16}$, a sample size of 51 per group was required. Allowing for a dropout of $30 \%$, we planned to enroll 140 children (70 in each group).

\section{Statistical analysis}

The normality of distributions of continuous data was assessed by KolmogorovSmirnov test. Normally distributed variables were descriptively presented as means and standard deviations. Non-normally distributed variables were descriptively pre- sented as medians and interquartile ranges. Pearson's chi-square test was used to compare categorical variables between groups. Normally distributed continuous variables were compared using Student's t-test whereas non-normally distributed variables were compared by Mann-Whitney U test. Statistical analysis was performed using SPSS version 16.0 for Windows (SPSS Inc., Chicago, IL). A P-value less than 0.05 was considered statistically significant.

\section{Results}

186 children were approached for participation in the study and 140 children accepted to be enrolled in the study (Figure 1). 70 children were randomized in to each study group. From the 140 children who were randomly assigned to the two groups, 10 children (4 in zinc group and 6 in placebo group) withdrew from the study within the first month due to concerns raised by the parents. Data from 130 children were included into the final analysis. From this group of 130 children, 2 children from the placebo group discontinued the study after the $3^{\text {rd }}$ month follow-up due to relocation to a different school. Data obtained until dis-

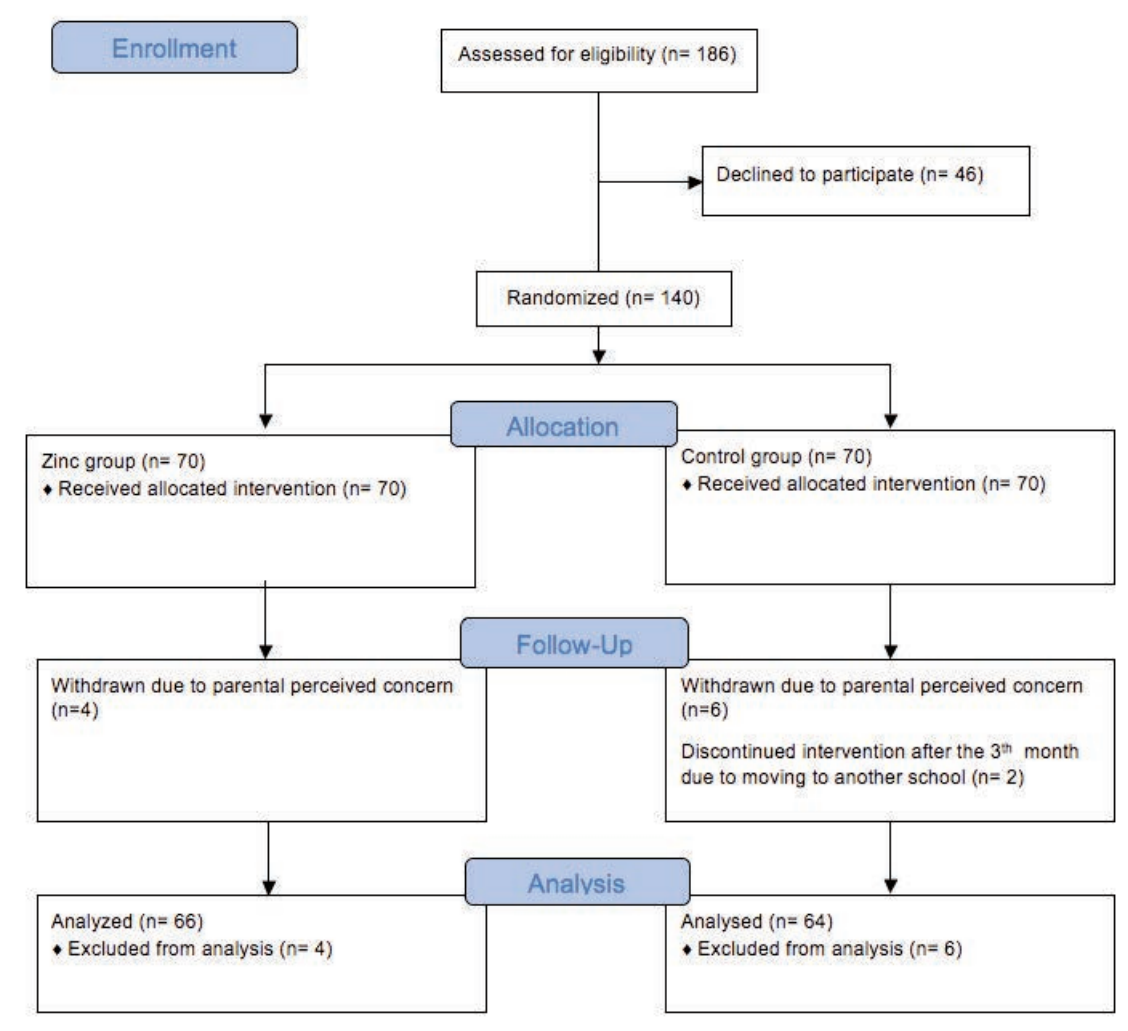

Figure 1. Study flow chart and enrolment. 
continuing the study were used for the final analysis. Compliance was good and similar in both groups with $82 \%$ of medication taken in zinc group and $84 \%$ by the placebo group $(\mathrm{P}=0.44)$.

Baseline demographic characteristics are shown in Table 1. Half of participants were boys with the mean age of 8.9 years (range: 5.7-12.8 years). There were no significant differences in terms of age, sex, weight, height, waist and hip circumferences, skin-fold thicknesses and body fat composition between the two groups.

Table 2 indicates the anthropometric data at the end of the study as well as the changes in each group during the study. At the end of the study, children in the zinc group were heavier than the children in the placebo group children but the differences of gross weight and weight $Z$-score were not significant. Gross weight gain after 6 months of study was $3.3 \pm 2.2 \mathrm{~kg}$ in zinc group and $3.2 \pm 2.2 \mathrm{~kg}$ in placebo group $(\mathrm{P}=0.865)$ and weight $\mathrm{Z}$ score increased in both groups by $0.22 \pm 0.34$ and $0.26 \pm 0.24$, respectively $(\mathrm{P}=0.501)$. At the end of study, children belonging to the zinc supplementation group were taller than placebo group children without a statistically significant difference $(133.3 \pm 13.6 \mathrm{~cm}$ and $130.1 \pm 11.6 \mathrm{~cm}$, respectively; $\mathrm{P}=0.153$ ). However, when we compared the height Zscore, zinc supplementation group were significantly taller than the placebo group (0.06 and -0.40 , respectively; $\mathrm{P}=0.013$ ). Gross height gain and height $\mathrm{z}$-score gain were significantly higher in the zinc group children than in the placebo group (height gain: $5.6 \pm 2.4 \mathrm{~cm}$ in Zinc group and $4.7 \pm 1.4 \mathrm{~cm}$ in placebo group; $\mathrm{P}=0.009$, height $\mathrm{Z}$-score gain: $0.45 \pm 0.37$ in Zinc group and $0.37 \pm 0.27$ in placebo group; $\mathrm{P}=0.048$ ). Other anthropometric parameters including waist and hip circumference, skinfold thickness and body fat composition increased from the baseline in both groups, however, there were no significant differences between study groups.

No major adverse events were reported for the study; three children in zinc group and 2 children in placebo group reported mild symptoms (nausea, mild abdominal pain and headache). These symptoms were resolved within a couple days, without any specific intervention and these children could complete the 6-months trial.

Table 1. Baseline characteristics and anthropometric data of the study population.

\begin{tabular}{lccc} 
& Zinc $(\mathrm{N}=66)$ & Placebo (N=64) & P value \\
Boys, $\mathrm{n}(\%)$ & $34(51.5)$ & $31(48.4)$ & 0.861 \\
Age, years & $8.9(2.1)$ & $8.8(2.0)$ & 0.821 \\
\hline Weight, $\mathrm{kg}$ & $29.1(12.2)$ & $27.1(9.3)$ & 0.295 \\
Height, $\mathrm{cm}$ & $127.7(12.7)$ & $125.4(11.3)$ & 0.277 \\
\hline WAZa & $0.25(1.48)$ & $-0.05(1.03)$ & 0.175 \\
HAZ & $-0.43(1.08)$ & $-0.77(0.93)$ & 0.053 \\
\hline Waist circumference, $\mathrm{cm}$ & $60.8(10.2)$ & $60.3(8.8)$ & 0.747 \\
Hip circumference, cm & $71.4(12.7)$ & $70.2(10.0)$ & 0.554 \\
\hline Mid arm circumference, cm & $19.1(4.5)$ & $18.8(3.4)$ & 0.646 \\
Biceps skinfold thickness, mmc & $4.5(5.0)$ & $4.5(3.5)$ & 0.790 \\
\hline Triceps skinfold thickness, mm & $8.9(4.4)$ & $8.4(3.8)$ & 0.544 \\
Subscapular skinfold thickness, mmc & $8.5(8.0)$ & $8.0(6.5)$ & 0.638 \\
\hline Supra-iliac skinfold thickness, mm & $15.0(9.1)$ & $13.2(7.5)$ & 0.227 \\
Body fat composition, $\%$ & $14.1(10.7)$ & $15.0(10.1)$ & 0.607 \\
\hline
\end{tabular}

Values are mean (SD). aWAZ= weight for age Z-score; bHAZ= height for age Z-score; cPresented as median (IQR)

Table 2. Anthropometric data at the end of study and changes of anthropometric from at the beginning of study in the study groups.

\begin{tabular}{|c|c|c|c|c|c|c|}
\hline & Zinc $(\mathrm{N}=66)$ & $\begin{array}{l}\text { End of study } \\
\text { Placebo }(\mathrm{N}=64)\end{array}$ & P value & Zinc $(\mathrm{N}=66)$ & $\begin{array}{l}\text { hange from Baselii } \\
\text { Placebo }(\mathrm{N}=64)\end{array}$ & P value \\
\hline Weight, kg & $32.4(13.5)$ & $30.3(10.8)$ & 0.336 & $3.3(2.2)$ & $3.2(2.2)$ & 0.865 \\
\hline WAZa & $0.47(1.51)$ & $0.20(1.14)$ & 0.252 & $0.22(0.34)$ & $0.26(0.24)$ & 0.501 \\
\hline Height, cm & $133.3(13.6)$ & $130.1(11.6)$ & 0.153 & $5.6(2.4)$ & $4.7(1.4)$ & 0.009 \\
\hline $\mathrm{HAZb}$ & $0.06(1.13)$ & $-0.40(0.95)$ & 0.013 & $0.45(0.37)$ & $0.37(0.27)$ & 0.048 \\
\hline Waist circumference, $\mathrm{cm}$ & $61.5(10.5)$ & $61.6(9.5)$ & 0.954 & $0.7(2.0)$ & $1.3(2.3)$ & 0.101 \\
\hline Hip circumference, $\mathrm{cm}$ & $72.6(11.0)$ & $71.5(9.9)$ & 0.564 & $1.2(7.3)$ & $1.3(4.5)$ & 0.905 \\
\hline Mid arm circumference, $\mathrm{cm}$ & $20.4(4.2)$ & $19.9(3.7)$ & 0.546 & $1.2(1.7)$ & $1.2(0.9)$ & 0.864 \\
\hline Biceps skinfold thickness, mmc & $6.0(4.3)$ & $6.0(4.0)$ & 0.359 & $1.0(2.0)$ & $1.0(2.0)$ & 0.973 \\
\hline Triceps skinfold thickness, mm & $10.2(4.2)$ & $10.2(3.7)$ & 0.992 & $1.4(3.0)$ & $1.8(3.0)$ & 0.393 \\
\hline Subscapular skinfold thickness, mmc & $10.0(7.5)$ & $10.0(10.0)$ & 0.710 & $1.0(3.0)$ & $2.0(3.8)$ & 0.395 \\
\hline Supra-iliac skinfold thickness, mm & $18.8(10.3)$ & $18.4(9.7)$ & 0.794 & $3.8(5.7)$ & $5.2(5.4)$ & 0.179 \\
\hline Body fat composition, $\%$ & $17.0(8.3)$ & $17.6(7.6)$ & 0.679 & $2.9(8.5)$ & $2.6(7.0)$ & 0.793 \\
\hline
\end{tabular}

Values are mean (SD). aWAZ= weight for age Z-score; bHAZ= height for age Z-score; cPresented as median (IQR) 


\section{Discussion and Conclusions}

The results of this double blind, randomized controlled trial indicated that zinc supplementation had a significant effect in improving the linear growth in Thai schoolaged children. We did not find any significant effects on the body weight or body composition (skin fold thickness and body circumferences) when compared to placebo.

Previous studies and meta-analyses on the effects of zinc supplementation in enhancing children growth had inconsistent outcomes. Brown et al. ${ }^{2,17}$ reported positive results on linear growth in 2 series of metaanalyses. However, Ramakrishnan et al. ${ }^{18}$ reported no benefit of increased height after zinc supplementation in children aged less than 5 years in a recent meta-analysis. However, most studies that included in the meta-analysis were studied in young aged children.

The present study confirmed the results on the efficacy of zinc supplementation for enhancing the growth of school-aged children of previous trials in selected populations. ${ }^{16,19}$ Ronaghy et al. ${ }^{19}$ reported that 40 $\mathrm{mg}$ of zinc supplement in form of zinc carbonate given with other nutrients (protein, lipid, vitamins and minerals) led to a significant increase in height and weight in Iranian malnourished school-aged boys. Interestingly, the improvements in body weight and height were not significant in the first 6 months of the study. However, at the end of the study after 12 months, the effect of zinc supplementation was significant in improving the body weight and height of the subjects. Our study lasted a total of 6 months and shows the positive effect of zinc in the first 6 months, in contrast to the findings of Ronaghy et al. ${ }^{19}$ The dosage of zinc in the present study was approximately 2-3 times of the recommended daily allowances, as used for treatment of zinc deficiency.20 The differences in results between our findings and those reported by Ronaghy et al. can be attributed to the different forms of zinc supplements used. We used a chelated zinc supplement which has a high absorption rate when compared to the zinc carbonate used in the Iranian study. Moreover this 1:2 metal to ligand ratio limit the inhibition of metal absorption by with dietary component and is not involved in oxidative reactions. ${ }^{1}$ Furthermore, the study by Ronaghy et al. only had male subjects and the study was conducted on malnourished children which growing in the controlled group that had caloric and other nutrients supplementation may enough to increase growth in the early stage (first 6 months) and the significant difference in growth will found in the later period after zinc storage expired. Another study that supported the efficacy of zinc supplementation in school-aged children was reported by Castillo-Duran et al. ${ }^{16}$ This group studied the effect of $10 \mathrm{mg}$ zinc sulphate supplementation on idiopathic short stature. This study, which again included only boys found a positive effect of zinc supplementation on the on gross height gain and height $\mathrm{z}$-score gain in pre-adolescent boys. Friis et al. ${ }^{21}$ found that zinc supplementation of 30 or $50 \mathrm{mg}$ had significant positive effects on gross weight gain, weight $\mathrm{z}$-score and arm muscle area at a shorter time period of 3 months. But this positive effect was not observed at a longer time period of 9 months in Zimbabwean pre-adolescent and adolescent. As the subjects were adolescent, the effect of zinc supplementation may not show a significant difference as the increase in height in adolescents depends on a number of factors including growth hormones and the differences in the age at the beginning of height spurt. Pre-adolescent age is less influenced by such factors and a comparison of height for age dependent Z-score change, as in our study, helps to limit the factors related to age dependence. In another study by Cavan et al.,22 a supplement of $10 \mathrm{mg} / \mathrm{d}$ of zinc as an amino acid chelate along with vitamins and minerals was compared to the control group supplemented with vitamins and minerals alone in Guatemalan children. The results of this study indicated that zinc supplements help to improve the body composition indices which included triceps skinfold and mid-arm circumference. No significant influences were observed on the height of the participants. This protocol is different in comparison to our protocol as our study does not include vitamin and mineral supplements. It has been generally observed that zinc supplementation in children less than 5 years of age has a significant positive effect on the on linear growth, especially when administered independently. ${ }^{23}$ However, the data on children in the preadolescence age, as in the case of our study, is insufficient to make any conclusions regarding the effects of zinc supplementation on the linear growth of children. We suggest for further study to make a strong recommendation.

In contrast to the above studies reporting a positive impact of zinc supplementation on growth in school aged children, there are some studies reporting an inefficacy of zinc supplementation. Mahloudji et al. 24 compared the efficacy of zinc and iron supplements to iron supplementation alone on growth enhancement in school-aged children. This study failed to demonstrate the efficacy of zinc and iron supplements on the growth or bone development. These suggest a counteractive effect of supplementation of two metals as the reason for inefficacy on the growth parameters, resulting in the negative outcomes. Evaluation of the plasma zinc concentrations by the authors also suggested that it was not a good indicator for zinc levels in the body as plasma zinc levels were not found to differ in the zinc supplemented group. The study underlines the importance of the study protocol and its impact on efficacy. Keeping in line with the findings of this study, we decided to adhere to a single supplementation for clarity of the results. Smith et al. ${ }^{25}$ studied the effect of zinc supplementation in aboriginal Australian children, partly comprising of adolescents and found no effect of zinc on the growth rates. The study calls for caution while interpreting the results of zinc supplementation as a part of the trial participants population were growth retarded with low plasma levels of zinc and might have other dietary deficiencies during the period of the study.

Apart from the above studies, there are two reports from Thailand: a study by Udomkesmalee et al.26 in 1992 and another one from Wasantwisut et al. 27 in 2006 on the effect of zinc supplementation in Thai children. Both studies have used a protocol different from the current study. Udomkesmalee et al.26 used zinc and/or vitamin A supplementation in school-aged children with low plasma levels of retinols and zinc. Their primary objective was to determine the biochemical and functional indices (visual restoration time) after vitamin A and zinc supplementation. The study concluded that zinc levels and visual function were improved after supplementation, however, they gave no details on the inefficacy of both supplements on growth. Wasantwisut et al. ${ }^{27}$ studied of zinc and/or iron supplementation in infants aged 4-6 months old. This age group is different from the age group of children targeted in our study. The results concluded that zinc supplements improved serum zinc levels without any effect on the growth.

The chemical form of zinc determines its bioavailability and hence the amount that is absorbed and assimilated from the intestines after digestion. Zinc is found as organic complexes with protein in meats, and as inorganic salts in plant foods. ${ }^{28,29}$ However, no isotopic studies have been carried out to assess the bioavailability of these different forms of zinc. Chelation of zinc to amino acids is known to improve the bioavailability of zinc as a result of increased solubility, ${ }^{30}$ in comparison to the inorganic forms of 
zinc as zinc sulfate. Zinc supplements sometimes result in symptoms including nausea and vomiting, epigastric pain, abdominal cramps, and diarrhea, ${ }^{31}$ and these effects were minimized when we used amino acid chelates in our study.

Our present study has some limitations as we did not analyze the plasma zinc levels, which could provide an understanding regarding the effect of supplementation on actual increases (if any) in blood. Plasma zinc measurement requires blood sampling (venepuncture) which is invasive and not well accepted by children and guardians. Thailand is classified by the International zinc Nutrition Consultative Group (IZiNCG) as a medium risk country of zinc deficiency in which the prevalence of zinc deficiency is estimated to be more than $40 \%$ in Thai population. ${ }^{11}$ Assessment of zinc status is difficult as it is associated with multiple metabolic functions and biochemical functions. Analysis of plasma zinc levels are also not regarded as a precise and sensitive method for zinc status. ${ }^{32,33}$ Plasma levels of zinc are expected to change several weeks after zinc depletion and therefore the plasma levels do not represent the levels during examination. ${ }^{34,35}$ Moreover, plasma zinc levels may not increase after zinc supplementation as has been previously reported. ${ }^{29}$ Moreover, differences in conditions such as food intake, endotoxemia, infection, steroids use, and plasma volume expansion may interfere with the interpretation of plasma zinc levels. ${ }^{33,36}$ Since there is a lack of reliable methods to assess zinc deficiency, it has been suggested that supplement trials should be used to define the extent of zinc deficiency. 37

Another limitation of our study is that we did not evaluate the dietary intake of the participants. Zinc absorption is inhibited by high intake of dietary phytates or concomitant intake with other minerals, thereby affecting zinc bioavailability. 38 However, all dietary assessment methods have a limitation in usage and may not accurate in assessment especially for children. ${ }^{39} \mathrm{We}$ controlled our study by blinded randomization and advised children not to take any test medications with the meals. Participants were also advised to avoid the intake of additional vitamins or mineral supplements during the study period.

As a recent meta-analysis of the effects of zinc supplementation showed a small but highly statistically significant, positive impact on children's linear growth and weight gain and a marginal effect on weight-for-height, ${ }^{17}$ a larger, multi-centric study at multiple end points would better clarify the exact role of zinc on the growth parameters of school aged children.
Interestingly, the meta analysis by Brown et al. 17 attributed the heterogeneity in the results of zinc supplementation to concurrent supplementation with iron and vitamin A. Trials with controls for each type of supplementation as well as their additive effect on the growth would be able to answer the presently open questions regarding zinc supplementation.

In conclusion, zinc supplementation improved linear growth (height) in healthy school-aged children without significant adverse event found. Zinc supplementation should be considered as a routine strategy to improve and reduce stunting in school-aged children in endemic area of zinc deficiency.

\section{References}

1. Prasad AS. Discovery of human zinc deficiency: its impact on human health and disease. Adv Nutr 2013;4:176-90.

2. Brown KH, Peerson JM, Rivera J, et al. Effect of supplemental zinc on the growth and serum zinc concentrations of prepubertal children: a meta-analysis of randomized controlled trials. Am J Clin Nutr 2002;75:1062-71.

3. Maret W, Sandstead HH. Zinc requirements and the risks and benefits of zinc supplementation. J Trace Elem Med Biol 2006;20:3-18.

4. Prasad AS. Zinc deficiency: its charac terization and treatment. Metal Ions Biol Syst 2004;41:103-37.

5. Aggarwal R, Sentz J, Miller MA. Role of zinc administration in prevention of childhood diarrhea and respiratory illnesses: a meta-analysis. Pediatrics 2007;119:1120-30.

6. Shakur MS, Malek MA, Bano N, et al. Serum and hair zinc in severely malnourished Bangladeshi children associated with or without acute lower respiratory infection. Indian J Pediatr 2009; 76:609-14.

7. Roth DE, Caulfield LE, Ezzati M, et al. Acute lower respiratory infections in childhood: opportunities for reducing the global burden through nutritional interventions. Bull World Health Organiz 2008;86:356-64.

8. Pinkaew S, Winichagoon P, Hurrell RF, et al. Extruded rice grains fortified with zinc, iron, and vitamin A increase zinc status of Thai school children when incorporated into a school lunch program. J Nutr 2013;143:362-8.

9. Akhtar S. Zinc status in South Asian populations - an update. Journal of health, population, and nutrition 2013; 31:139-49.

10. Thurlow RA, Winichagoon P,
Pongcharoen T, et al. Risk of zinc, iodine and other micronutrient deficiencies among school children in North East Thailand. Eur J Clin Nutr 2006; 60:623-32.

11. International Zinc Nutrition Consultative G, Brown KH, Rivera JA, et al. International Zinc Nutrition Consultative Group (IZiNCG) technical document \#1. Assessment of the risk of zinc deficiency in populations and options for its control. Food and nutrition bulletin 2004;25:S99-203.

12. Mayo-Wilson E, Imdad A, Junior J, et al. Preventive zinc supplementation for children, and the effect of additional iron: a systematic review and metaanalysis. BMJ Open 2014;4:e004647.

13. Khor GL, Misra S. Micronutrient interventions on cognitive performance of children aged 5-15 years in developing countries. Asia Pac J Clin Nutr 2012; 21:476-86.

14. Mayo-Wilson E, Junior JA, Imdad A, et al. Zinc supplementation for preventing mortality, morbidity, and growth failure in children aged 6 months to 12 years of age. The Cochrane database of systematic reviews 2014;5:CD009384.

15. Ministry of Public Health. Reference data for weight, height and indicators of nutritional status of Thai children. [cited 2017 Jan 30]. Available from: http://nutrition.anamai.moph.go.th/tem $\mathrm{p} / \mathrm{main} /$ view.php? group=5\&id=448.

16. Castillo-Duran C, Garcia H, Venegas $P$, et al. Zinc supplementation increases growth velocity of male children and adolescents with short stature. Acta Paediatr 1994;83:833-7.

17. Brown KH, Peerson JM, Baker SK, et al. Preventive zinc supplementation among infants, preschoolers, and older prepubertal children. Food Nutr Bull 2009;30:S12-40.

18. Ramakrishnan U, Nguyen P, Martorell R. Effects of micronutrients on growth of children under $5 \mathrm{y}$ of age: metaanalyses of single and multiple nutrient interventions. Am J Clin Nutr 2009;89: 191-203.

19. Ronaghy HA, Reinhold JG, Mahloudji $\mathrm{M}$, et al. Zinc supplementation of malnourished schoolboys in Iran: increased growth and other effects. Am J Clin Nutr 1974;27:112-21.

20. Saper RB, Rash R. Zinc: an essential micronutrient. Am Fam Physician 2009; 79:768-72.

21. Friis H, Ndhlovu P, Mduluza T, et al. The impact of zinc supplementation on growth and body composition: a randomized, controlled trial among rural Zimbabwean schoolchildren. Eur J Clin 
Nutr 1997;51:38-45.

22. Cavan KR, Gibson RS, Grazioso CF, et al. Growth and body composition of periurban Guatemalan children in relation to zinc status: a longitudinal zinc intervention trial. Am J Clin Nutr 1993;57:344-52.

23. Imdad A, Bhutta ZA. Effect of preventive zinc supplementation on linear growth in children under 5 years of age in developing countries: a meta-analysis of studies for input to the lives saved tool. BMC Public Health 2011;11:S22.

24. Mahloudji M, Reinhold JG, Haghshenass M, et al. Combined zinc and iron compared with iron supplementation of diets of 6- to 12-year old village schoolchildren in southern Iran. Am J Clin Nutr 1975;28:721-5.

25. Smith RM, King RA, Spargo RM, et al. Growth-retarded aboriginal children with low plasma zinc levels do not show a growth response to supplementary zinc. Lancet 1985;1:923-4.

26. Udomkesmalee E, Dhanamitta S, Sirisinha S, et al. Effect of vitamin A and zinc supplementation on the nutriture of children in Northeast Thailand. Am J Clin Nutr 1992;56:50-7.
27. Wasantwisut E, Winichagoon $P$, Chitchumroonchokchai $\mathrm{C}$, et al. Iron and zinc supplementation improved iron and zinc status, but not physical growth, of apparently healthy, breastfed infants in rural communities of northeast Thailand. J Nutr 2006;136: 2405-11.

28. Gibson RS. The role of diet- and hostrelated factors in nutrient bioavailability and thus in nutrient-based dietary requirement estimates. Food Nutr Bull 2007;28:S77-100

29. Solomons NW, Jacob RA, Pineda O, et al. Studies on the bioavailability of zinc in man. II. Absorption of zinc from organic and inorganic sources. J Lab Clin Med 1979;94:335-43.

30. Lonnerdal B. Dietary factors influencing zinc absorption. J Nutr 2000;130: 1378s-83s.

31. Brown MA, Thom JV, Orth GL, et al. Food Poisoning Involving Zinc Contamination. Arch Environ Health 1964;8:657-60.

32. King JC. Assessment of zinc status. J Nutr 1990;120:1474-9.

33. King JC. Zinc: an essential but elusive nutrient. Am J Clin Nutr 2011;94:679S-
$84 \mathrm{~S}$.

34. King JC, Shames DM, Lowe NM, et al. Effect of acute zinc depletion on zinc homeostasis and plasma zinc kinetics in men. Am J Clin Nutr 2001;74:116-24.

35. Baer MT, King JC, Tamura T, et al. Nitrogen utilization, enzyme activity, glucose intolerance and leukocyte chemotaxis in human experimental zinc depletion. Am J Clin Nutr 1985;41: 1220-35.

36. Hambidge M, Krebs N. Assessment of zinc status in man. Indian $\mathrm{J}$ Pediatr 1995;62:169-80.

37. Thacker N. Propagation of zinc for improving child health. Indian Pediatr 2007;44:489-90.

38. Troesch B, Jing H, Laillou A, et al. Absorption studies show that phytase from Aspergillus niger significantly increases iron and zinc bioavailability from phytate-rich foods. Food Nutr Bull 2013;34:S90-101.

39. Thompson FE, Subar AF, Loria CM, et al. Need for technological innovation in dietary assessment. J Am Diet Assoc 2010;110:48-51. 